\title{
\begin{tabular}{l|l} 
pcori $)$ & PATIENT-CENTERED OUTCOMES RESEARCH INSTITUTE \\
RESEARCH SUMMARY
\end{tabular}
}

PROJECT INFORMATION

March 2019

\section{Comparing Online Care with In-Person Care for Patients with Psoriasis}

Principal investigator

Organization

April W. Armstrong, MD, MPH

University of Southern California

\section{What was the research about?}

Psoriasis is a long-term skin disease that causes red, scaly patches. These patches may itch, hurt, or bleed. A dermatologist is a doctor trained to treat the skin. People with psoriasis can wait a long time or have to travel a long way to see a dermatologist. Online visits might help solve this problem.

In this study, the research team compared two ways of providing care to patients with psoriasis:

- Online visits with dermatologists

- In-person, face-to-face visits with dermatologists or primary care doctors

\section{What were the results?}

Online and in-person visits resulted in similar improvements in patients'

- Psoriasis

- Quality of life

- Symptoms of depression

Compared with patients who had in-person visits, patients who had online visits spent less time traveling to doctors' appointments. They also spent less time in doctors' offices.

\section{Who was in the study?}

The study included 296 adults from California and Colorado with psoriasis. Of these, 63 percent were white, 6 percent were Asian, 3 percent were black,
2 percent were Pacific Islander, 2 percent were American Indian/Alaska Native, and 24 percent were another race. Also, 34 percent were Latino. The average age was 49 , and 50 percent were women. To be in the study, patients needed internet access and a digital camera or a cell phone with a camera. Patients also needed a primary care doctor.

\section{What did the research team do?}

The research team assigned patients by chance to either online visits or in-person visits. To start an online visit with a dermatologist, the patient or the patient's primary care doctor

- Took photos of the patient's skin

- Put the photos and other information on a secure website

Within 48 hours, the dermatologist

- Recommended treatments to the patient and the primary care doctor through the website

- Wrote online prescriptions

- Provided patient education

If the patient or primary care doctor had questions, the dermatologist answered them online or by phone. Patients assigned to in-person visits saw a doctor of their choice as usual.

Patients filled out surveys before the study started and then every three months for one year. The surveys 
asked about skin health, quality of life, and depression.

The research team worked with people with psoriasis to plan the study and the website.

\section{What were the limits of the study?}

Patients' views about online or in-person visits might have affected how they thought about their treatment and changes in their health.

Future research could test online visits for more people and people from different parts of the country.
Researchers could also look at online visits for people with other long-term health problems.

\section{How can people use the results?}

Dermatologists, primary care doctors, and health clinics could use the results when planning ways for patients with psoriasis to get care.

To learn more about this project, visit www.pcori.org/Armstrong031. 